\title{
BMJ Open Nurse-led PrEP-RN clinic: a prospective cohort study exploring task-Shifting HIV prevention to public health nurses
}

\author{
Patrick O'Byrne (D) , Amanda Vandyk, Lauren Orser, Marlene Haines
}

To cite: O'Byrne P, Vandyk A, Orser L, et al. Nurse-led PrEP-RN clinic: a prospective cohort study exploring taskShifting HIV prevention to public health nurses. BMJ Open 2021;11:e040817. doi:10.1136/ bmjopen-2020-040817

- Prepublication history for this paper is available online. To view these files, please visit the journal online (http://dx.doi. org/10.1136/bmjopen-2020040817).

Received 01 June 2020 Revised 31 0ctober 2020 Accepted 11 December 2020

Check for updates

(C) Author(s) (or their employer(s)) 2021. Re-use permitted under CC BY-NC. No commercial re-use. See rights and permissions. Published by BMJ.

School of Nursing, University of Ottawa, Ottawa, Ontario, Canada

Correspondence to

Dr Patrick 0'Byrne;

pjobyrne@uottawa.ca

\section{ABSTRACT}

Objective To report the results of a nurse-led preexposure prophylaxis (PrEP) delivery service. Design This was a prospective cohort study conducted from 5 August 2018 to 4 March 2020. It involved manual chart review to collect data. Variables were described using frequencies and percentages and analysed using $\chi^{2}$ testing. Those significant in bivariate analysis were retained and entered into a binary multiple logistic regression. Hierarchical modelling was used, and only significant factors were retained.

Setting This study occurred in an urban public health unit and community-based sexually transmitted infection (STI) clinic in Ottawa, Canada.

Participants Of all persons who were diagnosed with a bacterial STI in Ottawa and everyone who presented to our STI clinic during the study period, there were 347 patients who met our high-risk criteria for PrEP; these criteria included patients who newly presented with any of the following: HIV contacts, diagnosed with a bacterial STI or single use of HIV PEP. Further, eligibility could be determined based on clinical judgement. Patients who met the foregoing criteria were appropriate for PrEP-RN, while lower-risk patients were referred to elsewhere. Of the 347 patients who met our high-risk criteria, $47 \%$ accepted and $53 \%$ declined. Of those who accepted, $80 \%$ selected PrEPregistered nurse (RN).

Primary and secondary outcome measures Uptake, acceptance, engagement and attrition factors of participants who obtained PrEP through PrEP-RN.

Findings $69 \%$ of participants who were eligible attended their intake PrEP-RN visit. $66 \%$ were retained in care. Half of participants continued PrEP and half were lost to follow-up. We found no significant differences in the uptake, acceptance, engagement and attrition factors of participants who accessed PrEP-RN regarding reason for referral, age, ethnicity, sexual orientation, annual income, education attainted, insurance status, if they have a primary care provider, presence or absence of depression or anxiety and evidence of newly acquired STI during the study period.

Conclusions Nurse-led PrEP is an appropriate strategy for PrEP delivery.

\section{INTRODUCTION}

HIV pre-exposure prophylaxis (PrEP) involves the daily use of HIV medications to prevent HIV acquisition by persons
Strengths and limitations of this study

The use of hierarchical modelling to identify the most relevant patient factors.

- A sufficiently robust sample size was used to answer our identified research objectives.

- All missing participant data were removed from our analyses.

who are HIV-negative. ${ }^{1}$ When used as prescribed, it can be up to $96 \%-99 \%$ effective in reducing HIV transmission. ${ }^{2}$ Traditionally, PrEP delivery involved direct in-person care from physicians in outpatient hospital or community-based clinics every 3 months. ${ }^{3}{ }^{4}$ With increasing demand for PrEP, other models of PrEP care have been created internationally, including (1) telemedicine models, ${ }^{5}$ where patients complete PrEP follow-up with providers via telephone or video conferencing; (2) community-based models, ${ }^{5}$ where PrEP services are administered by peers or community-based service workers; (3) self-management models, ${ }^{5}$ where patients independently monitor PrEP and report medication usage to their providers; and (4) home-based models, ${ }^{5}$ where patients complete at-home HIV and sexually transmitted infection (STI) self-testing and selfreport results to obtain PrEP.

Efforts to have nurses provide PrEP have also begun, albeit slowly. Sharma et a ${ }^{6}$ released a study protocol which outlines plans for STI clinic nurses to target PrEP at gay men. We, as well, have published clinical protocols, ${ }^{7}$ and a study in England ${ }^{8}$ showed that more patients could access PrEP when nurses are included in care, compared to when services were exclusively physician-delivered. Schmidt et al also implemented nurse-led PrEP clinics, wherein they had nurses 'screen, educate, clinically assess, order tests and manage results for initiation and follow-up visits, (p596) based on standing orders. These authors found that nurse-led PrEP was safe 
and increased provision of care in an accessible and appropriate way. ${ }^{9}$ Following medication initiation, studies have explored nurses' involvement with PrEP adherence and have demonstrated their effectiveness in optimising care. $^{10}$

While these models present solutions to increase uptake of, and access to, PrEP, aside from ours, they all involve physicians as the main provider. ${ }^{5}$ Even with the 'community-based' and 'nurse-led models ${ }^{5}$ that claim to involve alternate providers, physicians were ultimately responsible for monitoring, consultations, and prescriptions, thus rendering these clinics little other than an extension of the medical model of PrEP care.

With recent calls from the $\mathrm{WHO}^{2}$ for increased taskshifting of HIV prevention services to nurses, we sought to create a PrEP delivery model that transferred care from physicians solely to nurses. As part of this task-shifting initiative, we developed PrEP-registered nurse $(\mathrm{RN}),{ }^{7}$ the first PrEP service in Canada provided exclusively by RNs and nurse practitioners (NPs). In this paper, we briefly overview PrEP-RN and describe the outcomes of the first 19 months of patients who accessed care through this clinic. The research questions we focus on in this paper are: (1) What proportion of persons with HIV risk factors accept referral for PrEP from public health nurses?; (2) What does engagement in nurse-led PrEP care look like for persons with HIV risk factors? and (3) What factors predict PrEP attrition? We close this paper by asserting that task-shifting PrEP to nurses is appropriate, likely safe, and something to be expanded more broadly.

\section{PRE-EXPOSURE PROPHYLAXIS-RN}

In August 2018, we established PrEP-RN, which is a twopart PrEP referral ${ }^{11}$ and clinical service, ${ }^{7}$ operated by public health nurses and informed by the principles of task-shifting. The first part of PrEP-RN involved systematic PrEP referrals by nurses who work for our local public health unit for persons diagnosed with infectious syphilis or rectal gonorrhoea or chlamydia, or who were the sexual contacts of persons newly diagnosed with HIV. PrEP is also offered to persons who used post-exposure prophylaxis (PEP). Locally, chlamydia, gonorrhoea, syphilis and HIV are reportable, meaning that public health units must investigate persons diagnosed with these infections. Public health nurses complete this follow-up to ensure that appropriate testing, treatment, and referrals have occurred; a similar follow-up is completed with recent sexual partners ('contacts') of these individuals. For this study, we had public health nurses include PrEP referrals as part of their mandated follow-up for reportable STIs. Finally, our nurses were also able to refer patients to PrEP-RN based on clinical judgement. This allowed our nurses to refer patients to PrEP-RN when the foregoing high-risk criteria (table 1) were not fulfilled, but, clinically, the person was at high-risk for other reasons; for example, a patient who was always a contact of syphilis but never diagnosed (in that, our criteria for referral are based on the syphilis diagnosis), or someone who has other correlates of HIV acquisition, such as crystal meth use or group sex practices (table 1.). In short, nurses' clinical judgement allowed us to include persons who were at risk for HIV acquisition but did not fulfil our established criteria.

The second part of PrEP-RN is a PrEP clinic housed within our STI clinic, which is operated by RNs and NPs. ${ }^{12}$ Unique to PrEP-RN is that it includes task-shifting to nurses for referrals, clinical assessment, result interpretation and patient management-all tasks traditionally completed by physicians.

In summary, PrEP-RN has two parts: (1) assessments and referrals for PrEP by public health STI case managers and (2) the nurse-led clinic, with subsidised medication. To the best of our knowledge, this is the first systematic PrEP referral study involving a public health unit and the first solely nurse-led PrEP clinic in Canada. While other PrEP clinics claim to be nurse-led, most (if not all) are physician led, with nurses performing intake histories and specimen collection only. For example, a caveat to Schmidt $e t a l$ s 'nurse-led clinic' was that 'a doctor authorised under the study protocol must countersign any standing orders within 24 hours'. ${ }^{9} 596$ This clinic was, therefore, less about task-shifting and more about having nurses implement medical care.

\section{PrEP-RN clinical procedures}

For each participant, PrEP-RN nurses completed an intake visit and provided counselling on the medication, including how to take it and its efficacy and possible side effects. We used generic emtricitabine/tenofovir disoproxil fumarate for PrEP because it was the only medication approved for PrEP during the study period in Canada. Next, participants followed the Canadian PrEP guidelines ${ }^{13}$ and completed testing for HIV (using the fourth generation Abbott Architect), serum creatinine and STIs (genital, pharyngeal, rectal chlamydia/gonorrhoea,

Table 1 Indications for PrEP-RN referral

$\begin{array}{ll}\text { Objective criteria } & \text { New diagnosis of infectious syphilis } \\ & \text { More than one new rectal gonorrhoea or chlamydia diagnosis } \\ & \text { Contact with a person newly diagnosed with HIV } \\ \text { Clinical judgement } & \text { New post-exposure prophylaxis (PEP) use } \\ & \text { Variable based on patient characteristics }\end{array}$

PrEP-RN, pre-exposure prophylaxis-registered nurse. 
syphilis, hepatitis $\mathrm{B} / \mathrm{C}$ ), and performed needed vaccination (hepatitis A/B, human papillomavirus). Our nurses also assessed patients for signs and symptoms of HIV seroconversion, including mononucleosis-like symptoms, rash, and abdominal pain at each clinical visit.

RNs working under directives from NPs performed all testing and result interpretations and, provided there were no contraindications and laboratory findings were within target ranges, dispensed a 1-month supply of PrEP. This approach resulted in most participants obtaining their initiation prescription for PrEP approximately 7-10 days after their intake visit. (For details on our clinical procedures, including creatinine monitoring pathways, see O'Byrne $e t a l^{7}$ ) The same testing and clinical processes were repeated after 1 month and at subsequent 3 month visits, with the RN assessing medication use and side effects. The nurse also completed all relevant testing and result interpretations at that time. When a participant missed an appointment, our nurses would make up to two subsequent efforts to reschedule an appointment. We, therefore, considered participants who did not respond to two phone calls or text messages after a missed appointment as 'lost to follow-up'. After 12 months in the study, we referred all participants to a community physician or NP for ongoing PrEP.

For persons initiated on PEP, we offered direct PEP-toPrEP (PEP2PrEP) transitions. ${ }^{14}$ These patients were seen by week three of PEP initiation to complete PrEP counselling and baseline laboratory testing (HIV, creatinine) and, provided these results were within target ranges, were given a prescription to begin PrEP immediately after PEP. $^{14}$

PrEP medication was available at a pharmacy for purchase (for insured persons) or directly from the PrEP-RN clinic (for uninsured persons). Insurance included private coverage and public plans. In our jurisdiction, PrEP medication is free for all persons on social assistance and to those who are less than 25 years of age. It is also partially covered for persons with low income, but this form of coverage requires an application process. Persons without insurance could purchase PrEP medications directly from the PrEP-RN clinic at a cost of CAN $\$ 50$ per month.

\section{METHODS \\ Design}

This was a prospective cohort study conducted over 19 months (5 August 2018 to 4 March 2020). The COVID-19 pandemic interrupted data collection when the $\mathrm{WHO}^{15}$ declared a pandemic on 11 March 2020. All participants provided signed informed consent.

\section{Patient and public involvement}

We consulted community partner organisations (the AIDS Committee of Ottawa and Max Ottawa) regarding nurse-led PrEP and public health unit PrEP referrals. Community partners supported the approach taken for this study. The investigative team was independently responsible for the study design, data collection and analysis.

\section{Study setting}

During the study period, there were four local PrEP clinics: infectious disease physicians operated three and we implemented PrEP-RN. While anyone who wanted PrEP could access the infectious disease clinics (with some delay based on wait times), our clinic was for those who met our high-risk criteria through the public health unit and our STI clinic (table 1). The goal of PrEP-RN was to offer rapid PrEP initiations to persons with identified risk factors and those who our nurses identified as being at risk based on clinical judgement. The clinic was initiated on 5 August 2018 and continued to operate as of publication of this article, within our local STI clinic, which is located in downtown Ottawa, a city of 1 million people. This STI clinic has approximately 20000 patient visits per year for screening, testing, treatment and follow-up. Thus, for PrEP-RN, we selected a location that was familiar, easily accessible and community-based to provide PrEP. It was also the same location where we offer PEP. ${ }^{13}$ Notably, although our clinic continued from initiation through to the current period, there were temporary reductions in recruitment for the study during Spring 2020 due to the COVID-19 pandemic.

\section{Cohort}

Persons who were eligible for our study included anyone who was (1) prescribed PEP, (2) an HIV contact, (3) diagnosed with infectious syphilis or rectal gonorrhoea or chlamydia, or (4) classified by STI case management nurses in Ottawa as 'high risk' (table 1.) These criteria were based on HIV transmission estimates of $0.04 \%-1.38 \%$, ${ }^{13}$ the association between bacterial STIs and HIV acquisition, ${ }^{16}$ and a seroconversion rate of approximately $10 \%$ among participants in our PEP study. ${ }^{17}$ 'High risk' was based on nursing assessments. Thus, rather than focusing on gender, ethnicity and behavioural risk, our participant eligibility and recruitment criteria focused on objective clinical factors known to facilitate HIV acquisition. Our final cohort consisted of all patients who attended their intake visit within our PrEP-RN clinic.

\section{Data collection}

Data collection occurred in two ways. First, the STI case management nurses and the STI clinic nurses recorded on a standardised referral form all offers of PrEP to patients who qualified. Our nurses also collected participant information and risk factor data at baseline and at 1-month postinitiation, 4 months postinitiation and 7 months postinitiation, per Canadian PrEP guidelines. Measures of depression and anxiety symptoms were obtained using validated instruments (Patient Health Questionnaire-9 [PHQ-9], Generalized Anxiety Disorder-7 [GAD-7]). All data were collected by clinic nurses familiar with the study procedures through verbal questions and surveys, 
and recorded into a secure database designed for this purpose.

\section{Outcomes}

Within treatment status of PrEP use, we defined the outcome variable 'recruitment' as all active participants and those who were discharged to other PrEP clinics or lost to follow-up (ie, those who accepted PrEP and initiated and then continued or were referred or discontinued), compared with participants who were not seen (ie., never initiated on PrEP). Per the PrEP-RN clinical procedures, the term 'discharged' meant that participants accepted and proceeded with a referral to a community PrEP provider. The term discharged therefore signals ongoing PrEP use after a referral from PrEP-RN. In contrast, 'lost to follow-up' means participants were no longer using PrEP. To be 'lost to follow-up', per the PrEP-RN clinical procedures, a participant had to miss an appointment and then not respond to two further efforts by our clinic nurses to be contacted.

For retention, we looked at active and discharged participants compared with those lost to follow-up. We defined 'retention' as follows: a participant was continuing to use PrEP at a scheduled clinical visit. This means we did not consider retention as a static variable (in that we declared retention at a given point), but rather, accepted that retention could be lost at any point.

For adherence, we compared active and discharged participants to those who were lost to follow-up or not seen after referral. This approach allowed us to evaluate participants at different phases of PrEP and categorise them as engaged in care (whether in PrEP-RN or elsewhere) versus not engaged in PrEP care.

To explore participants' PrEP trajectories over the course of the study, we graphed visit completion over time. We also evaluated if participants followed scheduled visits, per the PrEP-RN clinical procedures. We defined follow-up as 'on protocol' if it aligned with the scheduled visits at intake, after 1 month and then every 3 months thereafter, and 'off protocol' as anything else. This variable helped us determine if participants followed extant PrEP guidelines.

\section{Data analysis}

We analysed the data using Microsoft Excel and the SPSS V.26.0. For PrEP-RN referrals, uptake and treatment status, we used descriptive statistics as appropriate. We also used descriptive statistics (frequency, percentage) for participant characteristics. All continuous variables were categorised according to predetermined cutoffs and categorical variables were collapsed as necessary to avoid small cell sizes. $\chi^{2}$ bivariate analyses were used to identify individual factors associated with the outcomes. We identified two variables with adequate cell size and significance $\mathrm{p}<0.1$, which we entered into a binary multiple logistic regression model using a hierarchical approach. From this, our plan was to retain only variables with significance at $\mathrm{p}<0.05$; however, neither variable demonstrated significance. Generally, missing data were minimal and

\begin{tabular}{lll}
\hline \multicolumn{2}{l}{ Table 2 PrEP referrals and uptake } \\
\hline $\begin{array}{l}\text { PrEP total Referrals } \\
\text { (N=347) }\end{array}$ & N/total (\%) \\
\hline Declined & & $185 / 347(53)$ \\
Accepted & & $162 / 347(47)$ \\
Choice=PrEP-RN* & Initiated & $129 / 162(80)$ \\
& Retrained & $89 / 129(69)$ \\
& & $58 / 89(69)$ \\
Treatment status & Discharged & $32 / 89(36)$ \\
& Active & $27 / 89(30)$ \\
Treatment completion & Lost to follow-up & $30 / 89(34)$ \\
& On-protocol† & $14 / 89(16)$ \\
& Off-protocol & $31 / 89(35)$ \\
Off-protocol completion & Too fast & $34 / 89(49)$ \\
time & Too slow & $0 / 31(0)$ \\
\hline
\end{tabular}

*Versus other available (physician-led) PrEP clinics. †'On-protocol' denotes that the participant wasusing PrEP and attending visits according to extant guidelines. 'Off protocol' signals that the participant was using PrEP but was attending visits that didnot align with extant guidelines.

PrEP-RN, pre-exposure prophylaxis-registered nurse.

random; therefore, we deleted cases cases with missing data listwise.

\section{RESULTS}

\section{Prep referrals, uptake and treatment status}

There were 347 eligible persons who met our clinical criteria for PrEP; 47\% ( $n=162)$ accepted PrEP and 53\% $(\mathrm{n}=185)$ declined. For the 162 persons who accepted PrEP, 33\% ( $=54)$ of these referrals were based on clinical judgement, 23\% $(n=37)$ were because of a new syphilis diagnosis, $17 \%(\mathrm{n}=27)$ were because of PEP initiation, $5 \%(n=8)$ were due to the identification of multiple rectal STIs, and $4 \%(n=6)$ were due to known HIV contacts. An final 19\% $(n=30)$ of referrals occurred because of multiple criteria (tables 2 and 3).

Of the 162 patients who accepted a PrEP referral, 129 $(80 \%)$ selected PrEP-RN. Ultimately, 89 (69\%) patients attended their PrEP-RN intake visit and were included in our cohort; $66 \%(n=59)$ of these participants were retained in care, with 27 still engaged in PrEP-RN, and 32 discharged to primary care or specialists for continuation of care. Three participants (5\%) discontinued PrEP due to fluctuations in kidney function that violated our pathways; creatinine values returned to normal after PrEP discontinuation and one of these participants has since restared PrEP without renal issues. No patient experienced any severe side effect listed in the product monograph (see figure $1 \mathrm{~A}, \mathrm{~B})$.

\section{Participant characteristics of those attending initial visit}

All participants identified as male and over three-quarters identified as gay. Most were white and 30\% were from other ethnic groups $(\mathrm{n}=8$ participants were Black, $\mathrm{n}=6$ 


\begin{tabular}{|c|c|c|}
\hline Factor & & $\begin{array}{l}\mathbf{N} \\
\text { (Valid \% } \\
\text { used) }\end{array}$ \\
\hline $\begin{array}{l}\text { Reason for referral } \\
(n=89)\end{array}$ & $\begin{array}{l}\text { Objective indicator } \\
\text { Clinical judgement }\end{array}$ & $\begin{array}{l}41(46) \\
48(54)\end{array}$ \\
\hline Age $(n=89)$ & $\begin{array}{l}<25 \text { years } \\
25+\text { years }\end{array}$ & $\begin{array}{l}18(20) \\
71(80)\end{array}$ \\
\hline $\begin{array}{l}\text { New STI during } \\
\text { treatment }(n=88)\end{array}$ & $\begin{array}{l}\text { No } \\
\text { Yes }\end{array}$ & $\begin{array}{l}70(79) \\
18(21)\end{array}$ \\
\hline Gender ( $n=89)$ & Male & 89 (100) \\
\hline $\begin{array}{l}\text { Sexual orientation } \\
(n=81)\end{array}$ & $\begin{array}{l}\text { Gay } \\
\text { Bisexual } \\
\text { Straight }\end{array}$ & $\begin{array}{l}62(77) \\
18(22) \\
1(1)\end{array}$ \\
\hline Education $(\mathrm{n}=80)$ & $\begin{array}{l}\text { Post-secondary } \\
\text { High school } \\
<\text { High school }\end{array}$ & $\begin{array}{l}58(73) \\
17(21) \\
5(6)\end{array}$ \\
\hline Income $(n=79)$ & $\begin{array}{l}<50 \mathrm{~K} / \text { year } \\
50 \mathrm{~K}+/ \text { year }\end{array}$ & $\begin{array}{l}43(54) \\
36(46)\end{array}$ \\
\hline Insurance $(n=88)$ & $\begin{array}{l}\text { Has insurance } \\
\text { Does not have } \\
\text { insurance }\end{array}$ & $\begin{array}{l}70(79) \\
18(21)\end{array}$ \\
\hline Ethnicity $(n=77)$ & $\begin{array}{l}\text { White } \\
\text { Non-white }\end{array}$ & $\begin{array}{l}54(70) \\
23(30)\end{array}$ \\
\hline $\begin{array}{l}\text { Primary healthcare } \\
\text { provider }(n=85)\end{array}$ & $\begin{array}{l}\text { Yes } \\
\text { No }\end{array}$ & $\begin{array}{l}47(55) \\
38(45)\end{array}$ \\
\hline $\begin{array}{l}\text { Symptoms of } \\
\text { depression }(n=67)\end{array}$ & $\begin{array}{l}\text { Yes } \\
\text { No }\end{array}$ & $\begin{array}{l}26(39) \\
41(61)\end{array}$ \\
\hline $\begin{array}{l}\text { Symptoms of anxiety } \\
\text { (67) }\end{array}$ & $\begin{array}{l}\text { Yes } \\
\text { No }\end{array}$ & $\begin{array}{l}21(31) \\
46(69)\end{array}$ \\
\hline
\end{tabular}

STI, sexually transmitted infection.

were of Middle Eastern decent, $\mathrm{n}=4$ were Asian). For education, three-quarters of participants held university/ college degrees/certificates, one-fifth completed high school and about 1 in 20 did not complete high school. Over half reported an annual income of less than CAN $\$ 50$ 000 , yet four-fifths had medication insurance. For STIs, one-fifth of participants were diagnosed with chlamydia, gonorrhoea or syphilis during the study. Two-fifths and one-third of participants met criteria for symptoms of depression and anxiety, respectively (table 3.) Finally, there were no statistically significant differences in the characteristics of participants who were included into the cohort because of nurses' clinical judgement versus an objective clinical indicator (table 4).

For HIV outcomes, no participant who initiated PrEP-RN was diagnosed with HIV. However, four persons who met our high-risk referral criteria (table 1) were diagnosed with HIV during the study period: two diagnoses were made based on PrEP intake laboratory results, and two diagnoses occurred in patients who delayed PrEP initiation (ie, all four were diagnosed before beginning PrEP). As such, 1.2\% $(n=4 / 347)$ of people meeting our

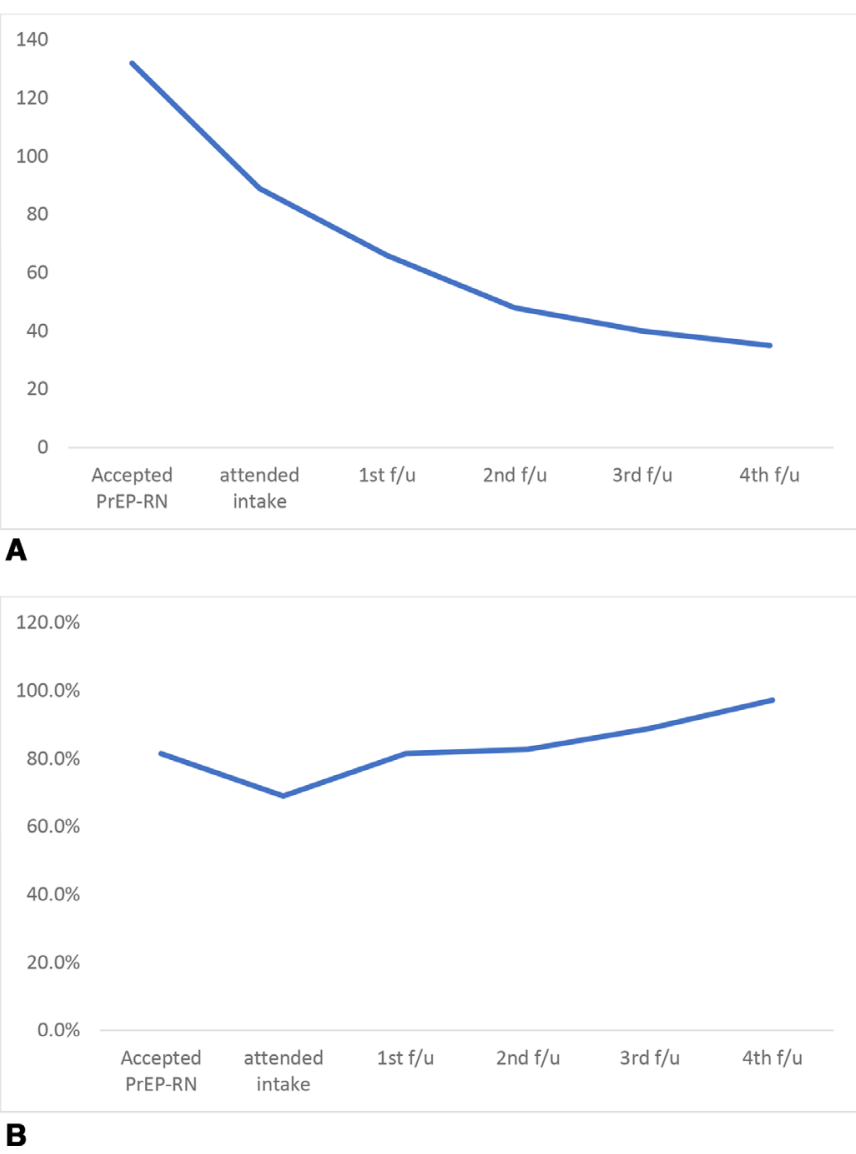

Figure 1 (A) PrEP-RN initiation and retention numbers. (B) f/u, follow-up; PrEP-RN initiation and retention rates. PrEP$\mathrm{RN}$, pre-exposure prophylaxis-registered nurse.

high-risk PrEP referral criteria ultimately acquired HIV during the study period.

\section{Treatment engagement and attrition}

As noted above, to evaluate engagement and attrition, we compared those who continued to use PrEP (whether still active in PrEP-RN or discharged to another PrEP clinic) versus discontinued (whether lost to follow-up or not

Table 4 Demographic variables of participants who initiated PreP because of objective indicator compared with clinical judgement

\begin{tabular}{lll}
\hline Demographic Variable & $\mathbf{X}^{2}$ & $\begin{array}{l}\mathbf{P} \\
\text { value }\end{array}$ \\
\hline $\begin{array}{l}\text { Age } \\
\text { (<25 years vs 25+ yrs) }\end{array}$ & 2.24 & 0.16 \\
$\begin{array}{l}\text { Sexual orientation } \\
\text { (gay vs bisexual/straight) }\end{array}$ & 0.33 & 0.59 \\
$\begin{array}{l}\text { Income } \\
\text { (<50 K/year vs 50 K+/year) }\end{array}$ & 2.76 & 0.11 \\
$\begin{array}{l}\text { Education } \\
\text { (high school of less vs postsecondary) }\end{array}$ & 0.03 & 1.00 \\
\hline \begin{tabular}{l} 
Ethnicity (white vs other ethnicities) \\
\hline
\end{tabular} & 0.22 & 0.80 \\
\hline
\end{tabular}

PrEP, pre-exposure prophylaxis-registered nurse. 
Table 5 Variables associated with retention in PrEP-RN (active/discharge vs lost to follow-up)

\begin{tabular}{lll}
\hline Variable & $\mathbf{X}^{2}$ & $\begin{array}{l}\mathbf{P} \\
\text { value }\end{array}$ \\
\hline $\begin{array}{l}\text { STI at treatment initiation } \\
\text { (yes vs no) }\end{array}$ & 2.96 & $0.09^{*}$ \\
\hline $\begin{array}{l}\text { STI diagnosed during tx } \\
\text { (yes vs no) }\end{array}$ & 0.23 & 0.63 \\
\hline $\begin{array}{l}\text { Age } \\
\text { (<25 years vs 25+ years) }\end{array}$ & 2.68 & $0.10^{*}$ \\
\hline $\begin{array}{l}\text { Sexual Orientation } \\
\text { (gay vs bisexual/straight) }\end{array}$ & 1.47 & 0.23 \\
\hline $\begin{array}{l}\text { Income } \\
\text { (<50 K/year vs 50K+/year) }\end{array}$ & 2.08 & 0.15 \\
\hline $\begin{array}{l}\text { Education } \\
\text { (high school of less vs post-secondary) }\end{array}$ & 0.01 & 0.95 \\
\hline $\begin{array}{l}\text { Insurance } \\
\text { (has insurance vs no insurance) }\end{array}$ & 0.76 & 0.38 \\
\hline $\begin{array}{l}\text { Ethnicity } \\
\text { (white vs non-white) }\end{array}$ & 1.72 & 0.19 \\
\hline $\begin{array}{l}\text { Primary care provider } \\
\text { (yes vs no) }\end{array}$ & 0.88 & 0.35 \\
\hline $\begin{array}{l}\text { Symptoms of depression } \\
\text { (yes vs no) }\end{array}$ & 0.82 & 0.37 \\
\hline $\begin{array}{l}\text { Symptoms of anxiety } \\
\text { (yes vs no) }\end{array}$ & 1.43 & 0.23 \\
\hline
\end{tabular}

*Not significant at $\mathrm{p}<0.05$ on further testing.

PrEP-RN, pre-exposure prophylaxis-registered nurse; STI, sexually transmitted infection.

seen after referral). Using this approach, half of participants $(n=45)$ completed treatment; the other half $(n=44)$ were lost to follow-up. Of the participants who continued PrEP, $69 \%(n=31 / 45)$ did so in a manner that contravened the expected trajectory as per the PrEP guidelines. (See table 1: on vs off-protocol.) In all 31 cases where participants received PrEP off-protocol (meaning their visit times and follow-up periods differed from extant guidelines), they progressed through the care faster than prescribed. That is, these participants were 'off protocol' because they attended more visits than would be expected based on the visit sequence recommended in current PrEP guidelines, as well as by the PrEP-RN clinical procedures: at intake, 1 month later, then every 3 months thereafter. These increased visits occurred because participants were diagnosed with STIs during PrEP visits (and thus had to return to clinic for treatment) and/or due to fluctuations in renal function test results.

While we expected certain factors to predict treatment attrition, we found no significant differences in reason for referral, age, ethnicity, sexual orientation, annual income, education attainted, insurance status, whether or not they have a primary care provider, presence or absence of symptoms of depression or anxiety, and evidence of newly acquired STI during the study period. (table 5.) Notably, most attrition occurred at the intake visit (figure 1A,B).

\section{DISCUSSION}

In the global context of efforts to increase access to PrEP through varying models of service delivery-and the $\mathrm{WHO}^{2}$ identifying HIV prevention as a key area for taskshifting-we established the first PrEP referral system and clinic in Canada that was wholly operated by nurses. In this paper, we present the first 19 months of PrEP-RN clinical operations, during which time we identified 347 eligible persons as high-risk for HIV, of whom about four-fifths chose PrEP-RN to receive PrEP. Of those who selected PrEP-RN, about two-thirds attended their intake appointment and two-thirds of those who attended intake were retained in care. We did not identify differences in the characteristics of patients who continued treatment compared with those who were lost to follow-up. Lastly, to the best of our knowledge, no PrEP-RN patient was diagnosed with HIV (after initiation) and only three discontinued PrEP based on our creatinine pathway-with one of these three participants having restarted without issue. These results raise a few points for discussion.

First, our results align with existing studies $^{6} 89$ on nurse-led PrEP and suggest that task-shifting PrEP care to nurses is appropriate. Thus far, there have not been any HIV diagnoses among PrEP-RN patients. We also only had to discontinue PrEP for three patients for medicationrelated kidney issues, which matches the rates of discontinuation for renal toxicity found in the initial and larger physician-run trials. ${ }^{18-20}$ This point about discontinuation is important because it helps validate our creatinine monitoring pathway, ${ }^{7}$ which could facilitate greater taskshifting to healthcare providers who may be less familiar with PrEP and renal function monitoring; for example, RNs or physicians who have not worked with renal function testing ever or in many years. This finding about our participants is also important because it supports previous analyses that found PrEP-RN to be a more cost-effective way to delivery PrEP. ${ }^{21}$ However, such economic analyses need to be redone, as over two-thirds of PrEP-RN patients engaged in more visits than listed in the guidelines. ${ }^{13}$ Comparisons now need to be made to determine the rate of follow-up visits in physician-run PrEP clinics to better determine the costs of offering PrEP. Lastly, our retention rate of $50.6 \%(45 / 89)$ matches that in the published literature about PrEP, ${ }^{22-25}$ and with larger reviews about medication adherence more generally, ${ }^{26}$ with about half of persons discontinuing any oral medication after about 12 months of use.

Further supporting task-shifting for PrEP is that, when given the choice, participants in our study overwhelmingly selected our nurse-led clinic as their preferred site to obtain PrEP. Concerns about receiving PrEP from non-physician prescribers did not materialise and instead were refuted by the fact that four-fifths of patients chose to receive PrEP from nurses. Lastly, regarding nurses' 
assessments skills in identifying who would benefit from PrEP, we did not identify any differences among participants to whom we offered PrEP based on established objective risk factors for HIV versus our nurses' clinical judgement. This highlights that there need not be concerns that nurses would either refer inappropriately or that nurses need standardised tools (eg, HIV incidence risk index for men who have sex with men [HIRI-MSM],${ }^{27}$ as their judgement matches the outcomes related to these tools. Recommendations for such screening tools are misguided and often suggested by non-nurses. ${ }^{27}$

As a second interesting point, our results highlight that, when PrEP is offered according to current clinical guidelines $^{13}$ (and not as a randomised controlled trial), recruitment and retention need improvement. The acceptance of PrEP and its continuation are low: only half of persons who met our high-risk criteria accepted a referral; only two-thirds of those who accepted PrEP-RN attended an intake visit; and only two-thirds of those who attended an intake visit were retained in care. (Results about why people decline PrEP are published elsewhere. ${ }^{28}$ ) Two interesting points on this matter were, first, that those who continued PrEP continued using it, and, second, that age demonstrated a trend toward significance. One reason why this might have occurred is that those who continue PrEP could simply agree with this intervention and thus continue PrEP because it aligns with their worldviews. These persons, conversely, may simply have a lower threshold for risk tolerance and use PrEP to manage this. Another possibility is that there may be tangible differences in the social perceptions of those $<25$ years old (versus older) regarding PrEP and its utility. Possibly, this could relate exclusively to the fact that, in our jurisdiction, those $<25$ years old can receive PrEP for free through a governmental medication plan, in which they are automatically enrolled. Uninhibited access to free PrEP, through an automatic plan and PrEP that is offered by nurses, may be the actual point of importance here. Further research needs to explore this point.

A final point of importance about our uptake rates is that, as our programme relied on nurses initiating conversations about PrEP with patients who met our high-risk criteria, even with only half of patients agreeing to PrEP, everyone who initiated PrEP through our project was someone who had not previously inquired about PrEP. Therefore, while the rates of acceptance were low, the actual number of those who used PrEP in the end needs to be compared with a zero, not to those who are eligible. None of the patients to whom we prescribed PrEP were previously using PrEP, nor had they inquired about it. All such referrals were initiated by our nurses, and thus, despite high levels of rejection, the acceptances constitute an increase in PrEP use.

\section{Limitations}

Our findings must be interpreted considering six main limitations. For one, our project occurred in a sociopolitical context where access to STI testing, treatment, and prevention services is fully funded by the government. If similar outcomes would occur in areas where people need private insurance to access care is unclear. Second, nearly all our participants reported same sex sexual partners, signalling that our findings might not apply in geographical areas where HIV is more common among persons with opposite sex sexual partners. Third, we only retained 89 persons in care, despite starting from a pool of 347 eligible persons. Thus, differences might have arisen if acceptance to initiate PrEP or retention in PrEP care increased. Fourth, some of the lack of identified associations may have arisen because not all participants provided data for certain variables. For example, about $25 \%$ of participants had not completed the anxiety screener. Fifth, our HIV diagnosis data are limited to patients who do testing using their given name in the province of Ontario. Those tested anonymously or in another province/country would not have an HIV diagnosis reported to our public health unit. More diagnoses among those who discontinued may have occurred. Sixth, we operate PrEP-RN as a clinical research study and ensure that all potential participants are aware of this fact before enrolment. As such, the rates of acceptance and non-acceptance may be an artefact of persons opting in or out of research.

\section{CLOSING REMARKS}

Notwithstanding certain limitations, our study highlights that task-shifting PrEP from physicians to nurses is a viable way to increase access to this HIV prevention intervention. That no harms occurred and that our discontinuation rates match the published literature reinforce that nurse-led PrEP is a viable option in need of further international development. In addition to a previous study showing that this approach is more cost effective, our current findings identified that PrEP-RN is a preferred option for patients to obtain PrEP. Considering that HIV rates continue unabated in many areas around the world, task-shifting HIV prevention-and specifically PrEP delivery-to nurses is one strategy to help curb ongoing HIV transmission. At this point, we feel there is sufficient evidence to scale-up such nurse-led interventions.

Correction notice This article has been corrected since it first published. The provenance and peer review statement has been included.

Collaborators Andree Bourgault; Marie-Odile Grayson; Megan Francoeur; Soo Ryun Lee.

Contributors PO was involved in all aspects of this study and paper. AV was involved in data analysis, paper drafting and finalisation. She was the lead for statistical analysis. LO was involved in all aspects of this study and paper. MH was involved in data analysis and paper drafting and finalisation.

Funding This work was supported by the Ontario HIV Treatment Network, grant number 1089. They had no influence on the study results and have not reviewed this paper or its findings.

Competing interests None declared.

Patient and public involvement Patients and/or the public were not involved in the design, or conduct, or reporting, or dissemination plans of this research.

Patient consent for publication Not required.

Ethics approval The research ethics boards at the University of Ottawa and at Ottawa Public Health approved this project (REB\#s: 149950 and 246-18).

Provenance and peer review Not commissioned; externally peer reviewed. 
Data availability statement Data are available on reasonable request. Data are available on request.

Open access This is an open access article distributed in accordance with the Creative Commons Attribution Non Commercial (CC BY-NC 4.0) license, which permits others to distribute, remix, adapt, build upon this work non-commercially, and license their derivative works on different terms, provided the original work is properly cited, appropriate credit is given, any changes made indicated, and the use is non-commercial. See: http://creativecommons.org/licenses/by-nc/4.0/.

ORCID iD

Patrick 0'Byrne http://orcid.org/0000-0002-0587-1409

\section{REFERENCES}

1 DHS T, Hull MW, Yoong D. Canadian guideline on HIV preexposure prophylaxis and nonoccupational postexposure prophylaxis. CMAJ 2017; 187:E1448-58

2 World Health Organization. Task shifting: global recommendations and guidelines, 2008. Available: https://www.who.int/healthsystems/ TTR-TaskShifting.pdf?ua=1

3 Sharma M, Wilton J, Senn H, et al. Preparing for PreP: perceptions and readiness of Canadian physicians for the implementation of HIV pre-exposure prophylaxis. PLoS One 2014;9:e105283.

4 Orser L, O’Byrne P. Implementing a nurse-led HIV pre-exposure prophylaxis service (PrEP-RN) in a public health unit STI clinic. HRO 2019;7:1-12.

5 Vanhamel J, Rotsaert A, Reyniers T, et al. The current landscape of pre-exposure prophylaxis service delivery models for HIV prevention: a scoping review. BMC Health Serv Res 2020;20:1-10.

6 Sharma M, Chris A, Chan A, et al. Decentralizing the delivery of HIV pre-exposure prophylaxis (PrEP) through family physicians and sexual health clinic nurses: a dissemination and implementation study protocol. BMC Health Serv Res 2018;18:513.

7 O'Byrne P, MacPherson P, Orser L, et al. PrEP-RN: clinical considerations and protocols for nurse-led PrEP. J Assoc Nurses AIDS Care 2019;30:301-11.

8 Girometti N, McCormack S, Devitt E, et al. Evolution of a preexposure prophylaxis (PrEP) service in a community-located sexual health clinic: Concise report of the PrEPxpress. Sex Health 2018;15:598-600.

9 Schmidt H-MA, Mclver R, Houghton R, et al. Nurse-Led preexposure prophylaxis: a non-traditional model to provide HIV prevention in a resource-constrained, pragmatic clinical trial. Sex Health 2018;15:595-7.

10 Mayer KH, Safren SA, Elsesser SA, et al. Optimizing pre-exposure antiretroviral prophylaxis adherence in men who have sex with men: Results of a pilot randomized controlled trial of "Life-Steps for PrEP". AIDS Behav 2017;21:1350-60.

11 O'Byrne P, Orser L, Jacob JD, et al. Responding to critiques of the Canadian PreP guidelines: increasing equitable access through a nurse-led active-offer PrEP service (PrEP-RN). Can J Hum Sex 2019;28:5-16.
12 O’Byrne P, Hollett M, Campbell B. A nurse practitioner leadership model for a sexual health clinic in Canada: staff feedback about implementation and operations. Nurse Leader 2020.

13 Tan DHS, Hull MW, Yoong D, et al. Canadian guideline on HIV pre-exposure prophylaxis and nonoccupational postexposure prophylaxis. CMAJ 2017;189:E1448-58.

14 O'Byrne P, Orser L, Vandyk A. Immediate PreP after PEP: results from an observational nurse-led PEP2PrEP study. J Int Assoc Provid AIDS Care 2020;19:232595822093976-7.

15 World Health Organization (WHO). Timeline: WHO's COVID-19 Response. Available: https://www.who.int/emergencies/diseases/ novel-coronavirus-2019/interactive-timeline\#! [Accessed August 28, 2020].

16 Barbee LA, Khosropour CM, Dombrowksi JC, et al. New human immunodeficiency virus diagnosis independently associated with rectal gonorrhea and Chlamydia in men who have sex with men. Sex Transm Dis 2017;44:385-9.

17 O'Byrne P, MacPherson P, Orser L. Nurse-Led HIV PEP program used by men at high risk for HIV seroconversion. $J$ Assoc Nurses AIDS Care 2018;29:550-9.

18 Solomon MM, Lama JR, Glidden DV, et al. Changes in renal function associated with oral emtricitabine/tenofovir disoproxil fumarate use for HIV pre-exposure prophylaxis. AIDS 2014;28:851-9.

19 Marcus JL, Hurley LB, Hare CB. Preexposure prophylaxis for HIV prevention in a large integrated health care system: adherence, renal safety, and discontinuation. JAIDS 2017;73:540-6.

20 Drak D, Barratt H, Templeton DJ, et al. Renal function and risk factors for renal disease for patients receiving HIV pre-exposure prophylaxis at an inner metropolitan health service. PLoS One 2019;14:e0210106.

21 O'Byrne P, Orser L, Jacob JD. The costs of HIV pre-exposure prophylaxis (PreP) care delivery: comparing specialists, primary care, and PrEP-RN. Sex Res Soc Pol 2019.

22 Chan PA, Mena L, Patel R, et al. Retention in care outcomes for HIV pre-exposure prophylaxis implementation programmes among men who have sex with men in three us cities. J Int AIDS Soc 2016;19:20903-8.

23 Hojilla JC, Vlahov D, Crouch P-C, et al. Hiv pre-exposure prophylaxis (PreP) uptake and retention among men who have sex with men in a community-based sexual health clinic. AIDS Behav 2018;22:1096-9.

24 Lankowski AJ, Bien-Gund $\mathrm{CH}$, Patel VV, et al. Prep in the real world: predictors of 6-month retention in a diverse urban cohort. AIDS Behav 2019;23:1797-802.

25 Zucker J, Carnevale C, Richards P, et al. Predictors of disengagement in care for individuals receiving pre-exposure prophylaxis (PreP). J Acquir Immune Defic Syndr 2019;81:e104-8.

26 Blaschke TF, Osterberg L, Vrijens B, et al. Adherence to medications: insights arising from studies on the unreliable link between prescribed and actual drug dosing histories. Annu Rev Pharmacol Toxicol 2012;52:275-301.

27 Wilton J, Mishra S, Tan DHS. Considerations for using the HIRI-MSM screening tool to identify MSM who would benefit most from PreP. $J$ Acquir Immune Defic Syndr 2017;76:e58-61.

28 O'Byrne P, Orser L, Haines M. (PrEP-RN) referrals: analysis of uptake rates and reasons for declining. AIDS \& Behaviour2019:1-9. 